\title{
Frequency Stop Band in an Air-Voided ZnO Photonic Crystal: A Dispersion Diagram Based Design
}

\author{
V. Chaturvedi ${ }^{a, *}$ And M. Kumar Roy ${ }^{b}$ \\ ${ }^{a}$ Department of Electronics\& Communication Engineering, Indian Institute of Information Technology, \\ Design and Manufacturing, Jabalpur, MP, 482001, India \\ ${ }^{b}$ Department of Natural Sciences, Indian Institute of Information Technology, \\ Design and Manufacturing, Jabalpur, MP, 482001, India
}

(Received November 20, 2015)

Electromagnetic excitation, inside small volumes, results in perturbations which play an important role in the validation of theoretical formulations. Efforts to catch a glimpse of the action inside of the small space, can aid better thin film designs. In the non-linear anisotropic regime the results of such interactions provide important insights into the bulk level behavior of matter. Using this approach, a zinc oxide $(\mathrm{ZnO})$ based photonic crystal is designed with spherical air voids. A Gaussian continuous wave excitation of the refractive index contrast $(\mathrm{ZnO}=1.9$ and Air =1) photon waveguide generated thus, is characterized for the redistribution of electromagnetic field. When, centered at a specific wavelength $(1.9 \mu \mathrm{m})$, the graph of the frequencies that can exist inside the crystal, is plotted against the limited $k$-space vector. The dispersion diagram that emerges shows a band of frequency states that cannot exist inside such a design. Physically this constitutes a $k$-space which is devoid of any detectable disturbances. Crystallographically, the reduced Brilluoin zone can be used to make a thin layer of $\mathrm{ZnO}$ that can act as a frequency stop layer, in a real multilayered photoelectric device.

DOI: $10.12693 /$ APhysPolA.130.683

PACS/topics: 01.65.+g, 01.70.+w, 41.20.Jb, 78.20.-e

\section{Introduction}

Natural excitations of the electromagnetic kind (for e.g., rays of the sun), inside a tightly bound, loosely held state of media such as a dense colloid, can be visualized as perturbations. Howsoever small, in nature, these disturbances have the capacity to change the steady state equilibrium of the medium. It falls upon the detection mechanism to sense the changes resulting from such an exposure. Physically, when the proportion of the lessdense-part is reduced dramatically (over time and space parameters) a condensed state of matter emerges. Slow heating, inside a muffle furnace, can be thought of as an example of the formation of such a state under "accelerated" natural conditions. This "artificial" process is akin to the removal of moisture and other gaseous form which results in the production of common salt. In comparison, the "real" process of natural salt formation is much slower. Research efforts focused towards deriving the fundamental properties of such matter-in-flux [1] states, seed, possibilities of a unifying theory.

To what extent have the perturbations disturbed the solid matter has been characterized as skin depth of that material. What happens just beyond the skin depth has been investigated. The initial theoretical [2] work gave way to experimental reports $[3-5]$ and culminated in a standard text [6]devoted to the computational modeling of control of light carrying photons. Photonics has thus

\footnotetext{
*corresponding author; e-mail: vinamrac@iiitdmj.ac.in
}

matured from just being the "science of photons" to a discipline in engineering [7]. In doing so it has opened new frontiers of research with diverse application possibilities.

However, in the design of real material system, the challenges associated with the light-matter interaction in the non-linear, inhomogeneous, anisotropic media remain. When applied to two important phenomena involving low dimensional electromagnetic exchanges of the light and matter, conventional optical phenomena have proved inadequate to explain certain dramatic results like (1) super lensing [8] and, (2) cloaking [9]. While one is associated with complete extinction of light, the other process is usually associated with an infinite expansion of the resulting fields. Using the example of zinc oxide (a metal-dielectric combination), here, we explain the basics of optical transparency (cloaking), by resolving the resulting electromagnetic field into a dispersion of frequency modes in the $k$-space .

\section{Design of model}

Properties of zinc oxide $(\mathrm{ZnO})$ have been well investigated [10]. Thin films made out of oxides of zinc are now poised to replace other oxide based layers because of the benign nature and transparent properties [11]. Photonic properties of $\mathrm{ZnO}$ are also under investigation because of the promising results of thin films grown using metalorganic-chemical-vapor deposition techniques [12]. Such results open the possibilities of engineering its band gap with a different approach.

Firstly, a computer based model [13] of $\mathrm{ZnO}$ based 3D photonic crystal with air voids was constructed using a 
face centered cubic (fcc) lattice arrangement. The air voids are visualized as a set of two spherical geometries, arranged in such a way that an elliptical waveguide results inside this lattice arrangement. A rough visualization of the geometry appears in Fig. 1. Noticeable skew in the supercell design consisting of the combined appearance of the lattice and the associated waveguide tries to mimic the arrangement of $\mathrm{ZnO}$ molecules vis a vis air voids thus creating an array of waveguides. To aid the readers imagination, in the later part of this paper we present a $2 \mathrm{D}$ projection of this $3 \mathrm{D}$ visualization, vide Fig. 2. The array of these waveguides behaves like "action center's", theoretically, generating eigenstates which support specific frequency modes.

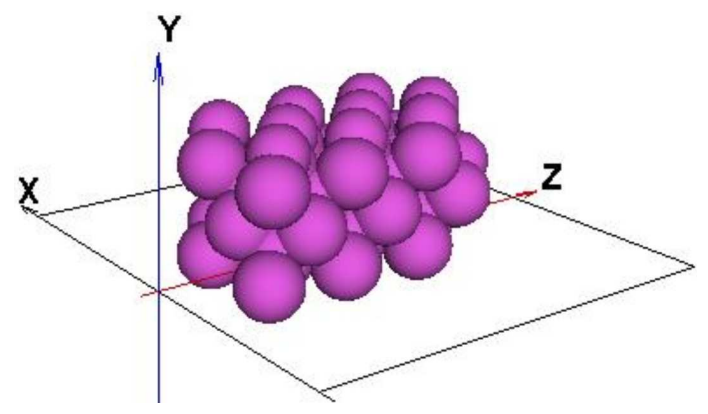

Fig. 1. A fcc crystal lattice with a block filling arrangement in a $3 \times 3$ configuration. Note the red arrow depicting the direction of exciting electromagnetic wave.

When compared to naturally occurring layers of a photonic crystal, the periodic arrangement of space voids [14] can assume shapes which provide "limited" guidance to interacting waves. In the formulation which builds on the difference in the refractive index between the air void and the surrounding media, physical shapes of the waveguides are overshadowed by waveguide shapes. This constitutes the basis of an effective medium in a theoretical formalism like ours. While the surrounding space, consisting of the substrate and the cladding layer, has the same refractive index as that of $\mathrm{ZnO}(n=2)$ [15], the two even sized spherical "balls" mimic the "air voids" $(n=1)$. This provides the necessary contrast needed in the computational electromagnetic solver based supercell. The constitution of such an array of contrasting waveguide is necessary towards setting up of an effective medium. Sufficient control on the design of the waveguiding properties of such a medium is exercised by the correct choice of exciting and observing parameters in the computational solver.

The parameters of the input electromagnetic excitation are set in the form of a Gaussian continuous wave. The direction of the input excitation is depicted by the red arrow in Fig. 1. Table I presents the additional details of the input excitation.

A plane wave, having a continuous Gaussian (GCW) profile centered at $1.9 \mu \mathrm{m}$, with a half width of $1.1 \times$ $10^{-14} \mathrm{~s}$ and a time offset of $5.5 \times 10^{-14} \mathrm{~s}$, excites previously designed structure with an amplitude power of
Input excitation details.

\begin{tabular}{c|c}
\hline \hline shape & Gaussian \\
nature & continuous wave \\
polarization & $Y$ direction \\
center wavelength & $1.9 \mu \mathrm{m}$ \\
half width & $1.1 \times 10^{-14} \mathrm{~s}$ \\
time offset & $5.5 \times 10^{-14} \mathrm{~s}$ \\
input amplitude power & $1 \mathrm{~V} / \mathrm{m}^{2}$ \\
field position & $0.2 \mu \mathrm{m}$ from structure
\end{tabular}

$1 \mathrm{~V} / \mathrm{m}^{2}$. This input field is positioned approximately $0.2 \mu \mathrm{m}$, away from the sample structure to ensure a continuous and evenly spread dose of the electromagnetic excitation to the $3 \mathrm{D}$ photonic crystal. Linear polarization along the $Y$ direction is maintained. The planar view of the overall excitation scheme along with the photonic crystal lattice arrangement is shown in Fig. 2. Notice the "green" circular shapes are the projections of the "violet" spheres (from Fig. 1). Also notice that there is "skewness" in the three lattice planes of the crystal. This is ensured by an uneven choice of parameters, at the time of designing the lattice simulation. Vector space $(1,1,1)$ is thus replaced by $(1,1,2)$.

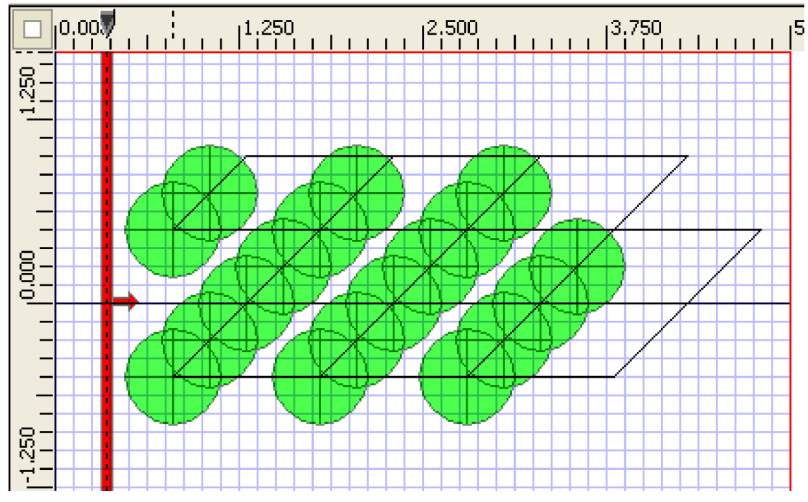

Fig. 2. Over all excitation scheme showing the input plane in red (on the left side of figure with a distinct arrow showing the direction of excitation). Note: the blue checkered background serves as an overall space with finite difference (FD), while the half width and time offset values, ensures overall the time domain (TD) based convergence.

Mathematically, a Gaussian profiled electromagnetic excitation, $E$, at an arbitrary point $x$ is expressed as

$$
A \exp \left(\frac{-\left(x-x_{0}\right)^{2}}{2 T^{2}}\right)
$$

where $A$ is the amplitude of excitation, $x_{0}$ is the center position, $T$ is the half width of the profile.

In general, in a Gaussian profile, the radius of the beam is the radial position at which the field amplitude and its intensity drops, to $1 / \mathrm{e}$ and $1 / \mathrm{e}^{2}$, respectively [16].

The Gaussian beam size or the Gaussian beam spot size is the beam diameter which is twice the beam radius. 
Suppose the beam size is noted as $a$, then we have

$$
\left.E\left(x=\frac{a}{2}\right)\right|_{x_{0}=0}=\exp \left(\frac{-a^{2}}{8 T}\right)
$$

This means that we have

$$
T=\frac{a}{2 \sqrt{2}} .
$$

This method is used by the solver to calculate the half width value.

Figure 3 shows the Gaussian profile of the electromagnetic excitation.
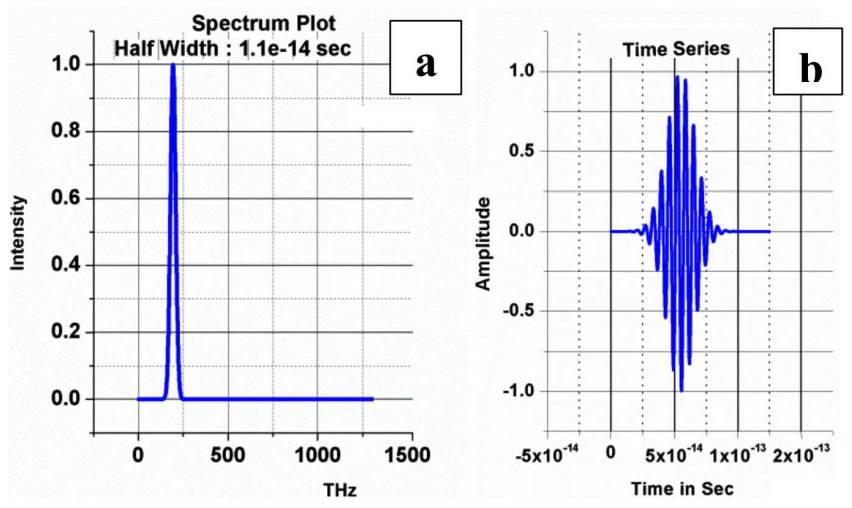

Fig. 3. Gaussian profile of the input excitation showing (a) time series plot, and (b) spectrum analysis. The time-offset value is set to $5.5 \times 10^{-14} \mathrm{~s}$ and the full wave half width value is $1.1 \times 10^{-14} \mathrm{~s}$.

The Maxwell equation in a transparent, time-invariant, source free, and non-magnetic medium can be written in the following form:

$$
\nabla \times \frac{1}{\varepsilon(\boldsymbol{r})} \nabla \times \boldsymbol{H}(\boldsymbol{r})=\frac{\omega^{2}}{c^{2}} \boldsymbol{H}(\boldsymbol{r})
$$

where $\varepsilon(\boldsymbol{r})$ - space dependent dielectric function, $c$ speed of light in vacuum, $\boldsymbol{H}(\boldsymbol{r})$ - magnetic field vector of frequency $\omega$ and time dependence $\mathrm{e}^{\mathrm{j} \omega t}$.

The master equation of the type (4), represents a Hermitian eigenvalue problem. This would not be applicable if the wave equations were derived in terms of the electric field.

The Bloch theorem states that due to infinite periodicity, the magnetic field will take the form

where

$$
\boldsymbol{H}(\boldsymbol{r})=\mathrm{e}^{\mathrm{j} \boldsymbol{k} \boldsymbol{r}} \boldsymbol{h}_{\boldsymbol{k}}(\boldsymbol{r})
$$

$$
\boldsymbol{h}_{\boldsymbol{k}}(\boldsymbol{r})=h_{\boldsymbol{k}}(\boldsymbol{r}+\boldsymbol{R})
$$

for all combinations of lattice vectors $\boldsymbol{R}$.

Thus, we end up with the master equation in the operator form

$$
(\nabla+\mathrm{j} \boldsymbol{k}) \times\left[\frac{1}{e(\boldsymbol{r})}(\nabla+\mathrm{j} \boldsymbol{k})\right] \times \boldsymbol{h}_{\boldsymbol{k}}=\frac{\omega^{2}}{c^{2}} \boldsymbol{h}_{\boldsymbol{k}} .
$$

This becomes the master equation that needs to be solved. The magnetic field is expanded in a finite basis of simple plane waves to transform this equation into a finite problem.

The result of solving the discretized problem (Eq. (7)) is the dispersion diagram which is a continuous plot between the eigenstate supported frequencies and the vector space represented by $\boldsymbol{k}$. This is usually plotted in the form of bands. Owing to the computational limitations we have restricted the calculations to a five band problem.

From a functional perspective, the appearance of a forbidden zone in the dispersion diagram signals the absence of any eigenstate which will support a set of frequencies that would appear otherwise, if the lattice arrangement inside the photonic crystal were to be different. This constitutes a frequency stop band. In a multilayered device setup, layers, made out of such an arrangement of crystal lattice based thin films, can be thought of as a pass layer for electrons but a stop layer for photons based conduction. Optical frequencies falling in the band gap range (actual value) would not be supported while remaining electrically conductive.

While evaluating the above parameters, caution needs to be exercised, in observing the redistribution of the electromagnetic field inside of the layered crystal lattice structure. An evenly (re)distributed field augments to the device performance under excitation.

Before the plot is actually implemented, the $\boldsymbol{k}$-vector path is defined in terms of reciprocal vectors . This is done so that the entire irreducible first Brillouin zone is scanned. Table II defines the parameters used in the $\boldsymbol{k}$-vector definition.

TABLE II

$G_{a}, G_{b}, G_{c}$ parameter definition for a irreducible first Brillouin zone.

\begin{tabular}{c|c|c|c|c}
\hline \hline$G_{a}$ & $G_{b}$ & $G_{c}$ & Div & \\
\hline 0 & 0.5 & 0.5 & 1 & $\mathrm{X}$ \\
0 & 0.625 & 0.375 & 3 & $\mathrm{U}$ \\
0 & 0.5 & 0 & 3 & $\mathrm{~L}$ \\
0 & 0 & 0 & 5 & $\mathrm{G}$ \\
0 & 0.5 & 0.5 & 5 & $\mathrm{X}$ \\
0.25 & 0.75 & 0.5 & 3 & $\mathrm{~W}$ \\
0.375 & 0.75 & 0.375 & 3 & $\mathrm{~K}$
\end{tabular}

\section{Results}

In our computation as the radius of the waveguide is increased and the origin of the lattice containing the second set of spherical waveguides is shifted (while retaining the fcc structure), eigenstates begin to appear in the $k$-vector space that do not support the existence of any of the dispersed frequency. These are the typical frequency stop bands that constitute the starting point of a device design of any photonic device. Thus the dispersion diagram of the photonic structure envisioned in Fig. 1 are plotted in Fig. 4. It should be noted that thicker bands get split into thinner smaller bands, once the waveguides are readjusted for the size and the planar symmetry within the lattice arrangement. These two parameters play an important role in the eventual tuning of the band gap within the $3 \mathrm{D}$ photonic structure. 


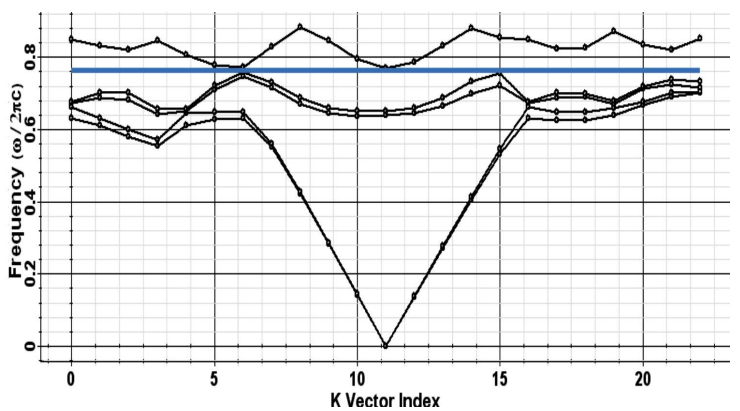

Fig. 4. Dispersion diagram plotted for a 5-band option showing a band gap (blue line) of 0.0122 between 0.7586 and 0.7708 .
Once the dispersion characteristics of the photonic structure are plotted, an important step towards studying the feasibility of the design is to check for the continuity of electromagnetic field within the computational space. This validates the boundary value conditions imposed by the Maxwell equations as discussed earlier. Using an analysis tool, $x, y$, and $z$ components of the electric and magnetic field are obtained. No particular discontinuity is observed in the simulation space. Some sample plots are presented in Fig. 5.
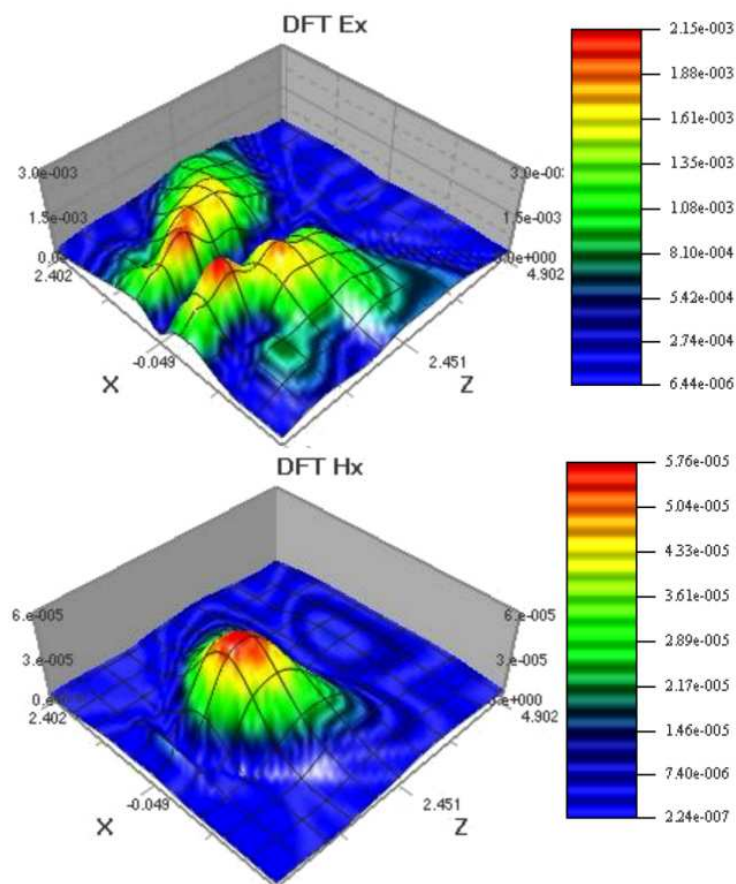
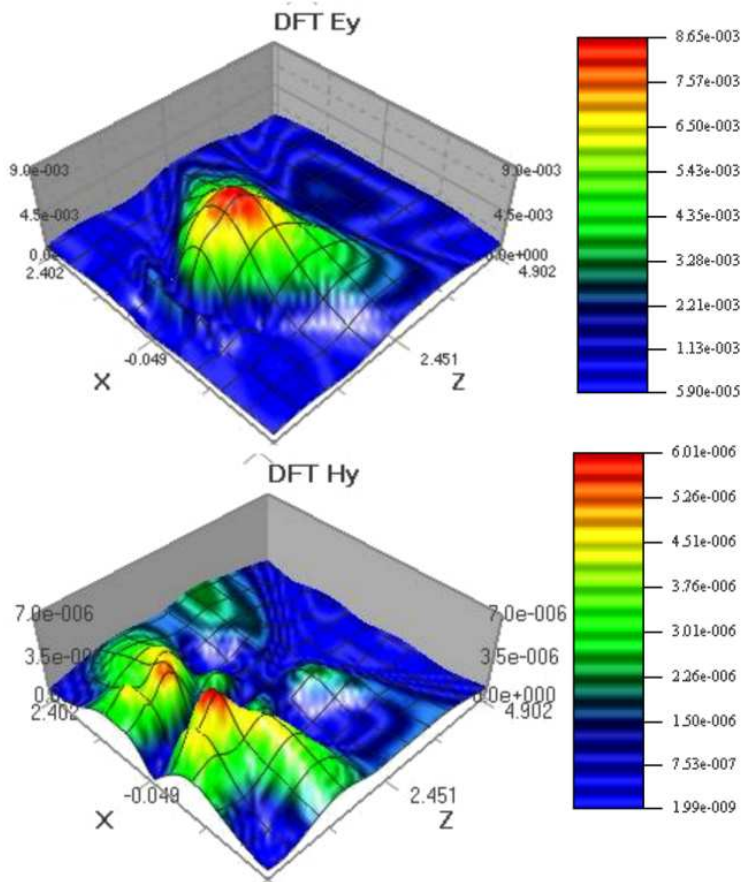

Fig. 5. Plots of $E_{x}$ and $H_{x}$ along with $E_{y}$ and $H_{y}$ in the simulation space. Notice sharp peaks close to the incident plane. Similarly, plots for the $z$-orientation of the redistributed electric and magnetic field are plotted.

Having checked the requirements of the continuity condition, we look for the power distribution in the simulation space. This is obtained by plotting the Poynting vector as shown in Fig. 6.

Sharp fluctuations in the vicinity of the lattice structure indicate an incident plane wave with a Gaussian continuous profile, perturbing the fragile balance of forces that exists prior to the excitation. This $3 \mathrm{D}$ photonic crystal structure of low refractive index contrast is capable of handling sharp fluctuation of the power intensity in the vicinity of the structure.

Use of plane wave excitation technique with a Gaussian continuous profile appears as powerful technique to check for the existence of frequency stop bands with a photonic crystal. The technique appears to be robust and repeatable at this stage of

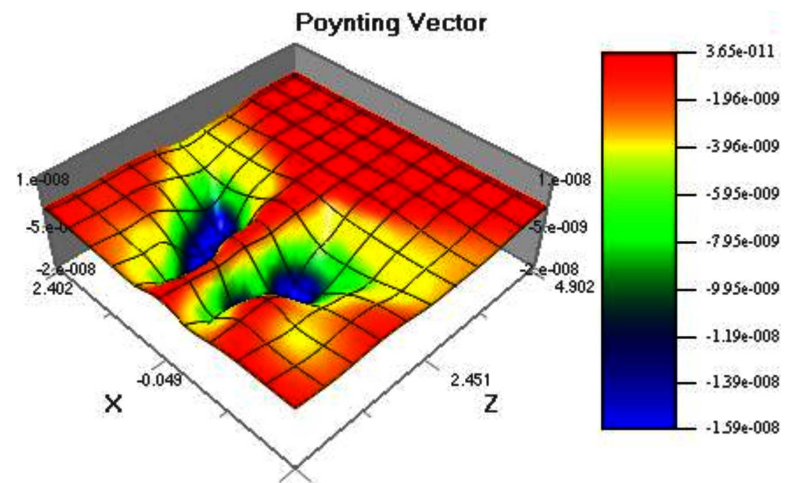

Fig. 6. Power distribution within the simulation space containing the $3 \mathrm{D}$ photonic crystal structure. 


\section{Acknowledgments}

This research was supported by the Ministry of Human Resources Development of the Government of India vide a scholarship grant \# 0910263 to one of the authors (V.C.).

\section{References}

[1] M.L. Gill, Phronesis 32, 1 (1987).

[2] V.G. Veselago, Sov. Phys. Usp. 10, 509 (1968).

[3] J.B. Pendry, Phys. Rev. Lett. 85, 18 (2000).

[4] E. Yablonovitch, Phys. Rev. Lett. 58, 2059 (1987).

[5] R.A. Shelby, D.R. Smith, S. Schultz, Science 292, 77 (2001).

[6] J.D. Joannopoulos, S.G. Johnson, J.N. Winn, R.D. Meade, Photonic Crystals: Molding the Flow of Light, 2nd ed., Princeton University Press, Princeton 2008.

[7] D.J. Lockwood, Silicon Photonics, Vol. 1, Ed. L. Pavesi, Springer Sci. \& Business Media, 2004, p. 3102.
[8] Meiling Liu, Maojin Yun, Feng Xia, Jian Liang, Proc. SPIE 8497, 849717 (2012).

[9] M. Selvanayagam, G.V. Eleftheriades, Phys. Rev. X 3, 041011 (2013).

[10] Z.L. Wang, J. Phys. Chem. B 104, 1153 (2000).

[11] Jin-Hong Lee, Kyung-Hee Ko, Byung-Ok Park, J. Cryst. Growth 247, 1 (2003).

[12] K. Minegishi, Y. Koiwai, Y. Kikuchi, K. Yano, M. Kasuga, A. Shimizu, Jpn. J. Appl. Phys. 36/2, 11A (1997).

[13] Product manual of OptiFDTD component designer.

[14] Jia-Zhe Liu, M.D.B. Charlton, Chung-Hsiang Lin, Kang-Yuan Lee, Chirenjeevi Krishnan, Meng-Chyi Wu, IEEE J. Quantum. Electron. 50, 314 (2014).

[15] Y.S. Park, J.R. Schneider, J. Appl. Phys. 39, 3049 (1968).

[16] R.W. Ziolkowski, Opt. Expr. 11, 662 (2003). 